\title{
LA LABOR LEXICOGRÁFICA BILINGÜE DE FRAY DOMINGO DE LOS SANTOS: VOCABULARIO DE LA LENGUA TAGALA
}

\section{THE BILINGUAL LEXICOGRAPHIC WORK OF FRAY DOMINGO DE LOS SANTOS: VOCABULARIO DE LA LENGUA TAGALA}

\author{
Marta Ortega Pérez \\ Universidad de Jaén \\ Universidad de Almería \\ mop00010@gmail.com
}

\begin{abstract}
RESUMEN
El presente artículo tiene como objetivo contribuir al estudio de la lexicografía bilingüe que tuvo lugar en Filipinas durante la colonización (ss. XVI-XIX). De este modo, se va a analizar la obra lexicográfica bilingüe de fray Domingo de los Santos. A continuación, se realizará un estudio exhaustivo con el fin de indagar las características principales de este diccionario, así como su intencionalidad didáctica. Para ello, se van a considerar la hiperestructura, la macroestructura y la microestructura. De este modo, se pretenden alcanzar los objetivos del trabajo: averiguar los modelos lexicográficos, ahondar en la estructura del diccionario, estudiar el material léxico, analizar el tratamiento lexicográfico de voces filipinas, americanas y españolas y, por último, descubrir la originalidad del autor como intérprete de la fe cristiana en lengua tagala.
\end{abstract}

Palabras clave: Filipinas, americanismos, léxico cristiano, definición, traducción

\begin{abstract}
This article aims to contribute to the study of bilingual lexicography that took place in the Philippines during colonization (16th-19th centuries). In this way, the bilingual lexicographical work of Fray Domingo de los Santos will be analysed. Down below, an exhaustive study will be carry out in order to investigate the main characteristics of this dictionary, as well as its didactic intentionality. Accordingly, the hyperstructure, the macrostructure and the microstructure will be considered. In this way, it is intended to achieve the objectives of the work: to find out the lexicographical models, to delve into the structure of the dictionary, to study the lexical material, to analyze the lexicographical treatment of Filipino, American and Spanish voices and, finally, to discover the originality of the author as an interpreter of the Christian faith in the Tagalog language.
\end{abstract}

Keywords: Philippines, Americanisms, Christian lexicon, definition, translation 


\section{INT'RODUCCIÓN}

El presente artículo tiene como objetivo presentar la obra lexicográfica de fray Domingo de los Santos (¿?, Cáceres - 1695, Mahayhay), esto es, su Vocabulario de la lengua tagala. Primera y segunda parte. En la primera se pone primero el castellano, y después el tagalo; y en la segunda, al contrario, que son las raíces simples con sus acentos, obra póstuma (1703).

El descubrimiento del Nuevo Mundo (1492) trajo consigo una necesidad inmediata de enseñanza de la lengua española, cuyo vehículo principal fue la propagación de la fe cristiana. De este modo, se crearon numerosas gramáticas y vocabularios. Así mismo, los religiosos de distintas órdenes se vieron obligados a tomar el papel de traductores. En consecuencia, el diccionario se convierte en una herramienta de instrucción social fundamental para la política lingüística de la época.

La producción lexicográfica bilingüe hispano-filipina, llevada a cabo durante la colonización y la evangelización de las provincias de Filipinas (ss. XVI-XIX), no ha sido tan estudiada como la realizada en el espacio americano. Dicho de otro modo, ha sido "uno de los capítulos más brillantes y olvidados de la historiografía lingüística hispánica" (García-Medall, 2007, p. 6).

En este trabajo, se va a analizar la obra mencionada para averiguar los modelos lexicográficos, con el fin de ahondar en la estructura del diccionario, el material léxico, la caracterización de las unidades léxicas y las definiciones. Asimismo, se atenderá la originalidad del autor como intérprete de la fe cristiana en lengua tagala.

Para conseguir los propósitos mencionados, se ha estudiado en profundidad la redacción del diccionario. Como se verá a continuación, el prefacio y la advertencia han revelado la intención cristiana del autor, asimismo, el análisis de la macroestructura y la microestructura de la parte castellano-tagala ha ayudado a descubrir cuáles fueron los posibles modelos lexicográficos. Cabe destacar que en esta obra no se analizará la segunda parte del diccionario, esto es, un listado de equivalencias tagalo-españolas que no refleja el quehacer lexicográfico del autor, además, no demuestra su singularidad como traductor de dos culturas totalmente distintas. 
Finalmente se presentará el tratamiento lexicográfico de las realidades filipinas, americanas y españolas, en cuanto a estas últimas el trabajo se centrará en las voces relacionadas con el léxico cristiano, ya que tiene mayor interés debido al objetivo evangelizador de la obra.

\section{LA LINGÜÍSTICA MISIONERA}

Esta investigación se inscribe dentro del campo de estudio denominado Lingüística Misionera, también llamada lingüística colonial o indigenista (Sueiro Justel, 2002). Se designa de esta forma al conjunto de trabajos, realizados por los misioneros, sobre las lenguas no europeas, cuyo objetivo era el aprendizaje y enseñanza de idiomas para facilitar la evangelización en los territorios colonizados entre los siglos XVI y XIX. Como indica K. Zimmermann (2004, p. 23):

Los lingüistas misioneros no tuvieron la meta de crear una teoría lingüística lo que les obligó a hacer un trabajo empírico sino su proyecto fue el de la enseñanza de lenguas. Los misioneros provienen de una tradición europea (latina y nebrijana [en el mundo hispánico]), aplican lo poco que existía y con el cambio de la meta de tradición europea se instrumentalizó con fines de política religiosa en un contexto de lenguas extranjeras.

Diferentes autores como Quilis (1982, 1997), Gutiérrez (1992), Fernández Rodríguez (2014), Sueiro Justel (2002, 2003), Moreno Moreno (2005, 2016) etc. señalan que la labor de los misioneros no fue fácil debido a la diversidad geográfica de aquellas islas, puesto que los habitantes estaban dispersos en diferentes llanuras, montes, etc., y, en consecuencia, no existía una organización de población. Por este motivo, los misioneros también se vieron obligados a establecer una disposición de los habitantes dentro de aquellas tierras para que fuera más fácil la enseñanza de la doctrina cristiana.

Por lo tanto, existían dos problemas importantes para llevar a cabo la evangelización, por un lado, la geografía y, por otro, la distribución desafortunada de los habitantes de Filipinas.

No hay duda de la ardua labor de los misioneros durante esta etapa de descubrimientos donde la política de la corona exigía la evangelización de los nuevos pueblos y la enseñanza de la lengua española. En este sentido, cabe destacar que, en Filipinas, a pesar de que en un principio los clérigos decidieron predicar la palabra de 
dios en castellano, este método no era resultante para su objetivo. Por lo tanto, se dieron cuenta de la necesidad de aprender las lenguas indígenas para poder predicar con exactitud y sin divagaciones, es decir, al conocer la cultura y la lengua del otro, pudieron codificar, en cierta medida, las lenguas y realizar una serie de obras que los ayudarían tanto a aprender las lenguas indígenas como a enseñar el español y, en consecuencia, el evangelio.

Los estudios sobre la Historia de la Lingüística Misionera ponen en boga el quehacer lingüístico de los clérigos. Estos religiosos tomaron el papel de lingüistas y crearon una metodología exhaustiva para realizar transcripciones fonético-fonológicas, recopilar datos morfológicos, sintácticos, así como semánticos. Todas estas obras sirvieron de partida para la lingüística descriptiva y estructural. De hecho, autores como Hervás y Panduro y Humboldt integraron en sus teorías algunos de los resultados de las obras realizadas por los misioneros (Zimmermann, 2004).

Cabe destacar que este tipo de disciplina se desarrolló en un contexto históricocultural de una sociedad monoteísta que temía por la herejía del mundo e intentaba convertir al cristianismo a todo aquel que procedía de otra religión.

\section{DOMINGO DE LOS SANTOS: VOCABULARIO DE LA LENGUA TAGALA}

Según los datos recogidos por la Biblioteca Virtual de Filología Española, dirigida por Manuel Alvar Ezquerra, fray Domingo de los Santos nació en Torrejoncillo de Extremadura (Cáceres). El autor del diccionario pertenecía a la orden franciscana, aunque, erróneamente se le ha considerado alguna vez dominico (BVFE $\left.{ }^{1}\right)$. Además, fue seguidor de la vía reformista encabezada por el también extremeño y franciscano San Pedro de Alcántara (1499-1562) y llegó a Filipinas en 1641. Se dedicó a la labor evangelizadora en diversas localidades del archipiélago y ocupó el cargo de definidor en 1687. Finalmente, murió en 1695 en Mahayhay.

La BVFE también indica que fue gran conocedor, al parecer, de la lengua tagala, de hecho, en el diccionario encontramos muchas explicaciones sobre la cultura de los hablantes del tagalo. Según Gómez Platero (1880) fue el más versado de su tiempo en

${ }^{1}$ Biblioteca Virtual de la Filología Española (https://www.bvfe.es/) 
la lengua tagalog y advierte que escribió una gramática que no se consiguió imprimir. No obstante, se conservan algunas hojas de este arte que debió de circular dentro de la comunidad misionera del archipiélago e influyó mucho en autores posteriores, como en Sebastián de Totanés, O. F. M. (1687-1748) (BVFE).

El diccionario fue publicado póstumamente en Tayabas en 1703, si bien la redacción original es de 1688 y fue reeditado dos veces, en 1794 y en 1835 . En este trabajo se va a analizar la edición de 1794, puesto que no ha sido posible contar con la publicación de 1703.

En el Vocabulario de la lengua tagala se diferencian cuatro partes: el prefacio, la advertencia al lector, el diccionario a dos columnas en castellano con su traducción en tagalo y el cuarto, un listado de equivalencias tagalo-castellanas.

En cuanto al prefacio, cabe destacar que se trata de una dedicatoria, en primer lugar, a la provincia de san Gregorio de Religiosos Descalzos en la que se señala que esta obra se ha escrito para devolver el favor tanto de la ciudad como de la orden franciscana (Domingo de los Santos, 1794 [1703]: prefacio). Igualmente, indica que esta obra, a pesar de haber sido causa de desvelo, la realizó gracias a la enseñanza que le dio la tierra ${ }^{2}$. Asimismo, el autor ofrece información sobre la labor misionera en la provincia y hace mención a la enseñanza y aprendizaje de la lengua tagala.

En la advertencia al lector da información sobre la elaboración de vocabularios de la época, ya que anuncia que fue necesario este vocabulario debido a que no existían muchos impresos y a la dificultad y gasto en traducirlos. Asimismo, apunta que los existentes estaban anticuados y otros no se conocían. En este apartado habla de las gramáticas que el autor ha usado para realizar el diccionario. Los gramáticos que cita son: el padre fray Andrés Verdugo de la orden de san Agustín y el padre fray Francisco de Joseph de la orden de Nuestro Padre santo Domingo. Además, indica que las voces las ha recogido del vocabulario de 1613 de Pedro de san Buenaventura y de un manuscrito de f'ray Francisco de san Antonio, ambos religiosos de la orden de san Francisco. Por último, explica detalladamente el significado de cada abreviatura que

\footnotetext{
2 Provincia de san Gregorio Magno de Religiosos Descalzos de la Orden de San Francisco.
} 
aparece en el cuerpo del diccionario. Todas estas marcas son explicaciones gramaticales, fonéticas, remisiones, marcas diatópicas, etc.

La obra lexicográfica estudiada tiene un objetivo muy claro, enseñar a los aprendices misioneros a predicar la palabra del evangelio. Por lo tanto, la subjetividad abunda en el diccionario constantemente. En cuanto a la macroestructura, cabe destacar que aparece un número considerable de voces cristianas. Asimismo, en los distintos artículos lexicográficos, se han localizado diversas marcas ideológicas que nos indican que el Vocabulario es un instrumento de predicación que ayuda a la enseñanza de la propagación de la fe cristiana.

\subsection{LOS MODELOS LEXICOGRÁFICOS DEL VOCABULARIO}

Después de este análisis sobre la estructura del diccionario, se van a indagar los modelos lexicográficos de esta obra y los referentes que el autor leyó para realizar su Vocabulario.

Domingo de los Santos subraya que siguió a fray Pedro de San Buenaventura, como modelo lexicógrafo. En este punto cabe destacar que san Buenaventura redactó el primer diccionario hispano-tagalo: Vocabulario de la lengua tagala, el romance castellano puesto primero. Seguramente haya servido como referente para otros autores que trataron de describir esta lengua y ha sido considerada como una joya de la literatura por dos motivos: obra incunable filipina y por ser el primer diccionario de una lengua filipina impreso por los misioneros españoles.

Después de la comparación de las dos obras, se observa que la disposición del diccionario es la misma, es decir, es un diccionario bidireccional. En primer lugar, aparece el diccionario español-tagalo y en segundo lugar el diccionario tagalocastellano. Cabe advertir que una característica de los vocabularios hispano filipinos, como ocurre en el primer vocabulario impreso de Pedro de San Buenaventura (1613), según García-Medall (2007, p. 11) y se ha podido comprobar, la parte castellano-tagala comprende un $85 \%$ de la obra, mientras que la parte tagalo-española es un glosario de correspondencias sin ninguna información fonética, semántica o gramatical. Lo mismo ocurre con este Vocabulario, lo cual no cabe duda que en cuanto a la selección léxica y elaboración de diccionario siguió la obra de Buenaventura. 
En este trabajo se va a tratar la primera parte castellano-tagala del Vocabulario, puesto que como se ha indicado anteriormente, constituye una parte considerable de la obra y es la más representativa en cuanto a cuestiones de lexicografía.

Dentro de los modelos lexicográficos, este autor también habla de un manuscrito de fray Francisco de san Antonio. No obstante, este manuscrito está perdido y no se ha podido comparar.

En cuanto a la ordenación lexicográfica, cabe destacar que, según García-Medall (2007, p. 11), existía una codificación de traducción en las órdenes religiosas. Mientras que los franciscanos, en los diccionarios bilingües, ponían en primer lugar las voces castellanas y sus equivalentes tagalos, los agustinos seleccionaban en primer lugar las voces filipinas y después los equivalentes castellanos. Por lo tanto, había una doble tradición lexicográfica. Siguiendo a Moreno Moreno (2016, p. 219), la ordenación español-filipina se debía a una cuestión pedagógica que partía del Vocabulario nebrisense y que en América se reprodujo de forma temprana el Vocabulario en lengua castellana y mexicana (1555) de Alonso de Molina. Este modelo de ordenación lexicográfica por lo tanto tenía una tradición que fue seguida por San Buenaventura (1613) y la continúa Domingo de los Santos (1794 [1703]) en la que se aprecia que la parte español-lengua filipina prima en la obra.

Cabe destacar que Domingo de los Santos también indica las gramáticas que ha usado para poder realizar el diccionario: por un lado, la obra del padre fray Andrés Verdugo de la Orden de san Agustín y, por otro, la gramática del padre fray Francisco de Joseph de la orden de Nuestro Padre santo Domingo. No obstante, en este trabajo no se van a estudiar los aspectos gramaticales que aparecen en los artículos lexicográficos por diversas razones:

1) El autor enumera hasta "diecisiete especies de verbos activos y pasivos (1794 [1703]: advertencia)", de la obra del padre fray Andrés Verdugo de la Orden de san Agustín. Como indica García-Medall (2007, p. 12), la existencia de tantas formas gramaticales es "un hecho descabellado, contrario a las gramáticas de las lenguas filipinas y desastroso desde la perspectiva pedagógica". 
2) Existen gramáticos, como fray Francisco de san José de la orden franciscana, que realizaron grandes esfuerzos para reducir las reglas tagalas (Quilis, 1982), debido a la dificultad que el gran número de normas gramaticales causaba para la enseñanza y aprendizaje.

Como conclusión de este apartado, cabe decir que, según García-Medall (2004, 2007), el primer vocabulario bilingüe en América para la lengua náhuatl o mexicana (Alonso de Molina, 1555) fue un referente en cuanto a la selección léxica de las obras lexicográficas. Esta obra sigue la ordenación de la tradición nebrisense y que es seguida en Filipinas, como se puede ver en el primer vocabulario bilingüe hispano-filipino de san Buenaventura y que ha sido seguido por Domingo de los Santos [1794 (1703)].

Además, la obra lexicográfica estudiada pertenece al periodo clásico de la lexicografía hispana-filipina (1610-1765). Cabe destacar que en cuanto a la historia lexicográfica bilingüe hispano-filipina este periodo representa el quehacer lexicográfico de las lenguas más importantes del norte y del centro del archipiélago (García-Medall 2007). Estas lenguas son: el bisaya, el tagalo, el pampango o el ilocano. Estas obras fueron un fracaso en el sentido de la representación gramatical si son comparadas con las obras gramaticales que se realizaron por otros religiosos: Blancas de San José (1610) para el tagalo, el padre Francisco López (1628) para el ilocano, el padre Méntrida (1818 [1618]), para el bisaya, el padre Andrés López (1621) para el pangasinán y el padre Benavente (1699) para el pampango.

La importancia de la lexicografía bilingüe de este periodo se encuentra en la información léxica y pragmática que aportan. A esto hay que añadir que en este sentido se parecen a los diccionarios coetáneos realizados en el continente americano.

\subsection{EL CORPUS LEXICOGRÁFICO}

Domingo de los Santos conocía bien su misión como predicador. Por lo tanto, elaboró su obra como instrumento para la enseñanza del español a través de la doctrina cristiana. De este modo, vamos a encontrar alrededor de 180 voces propias del léxico cristiano. Todas ellas han tenido un proceso de traducción diferente debido a la naturaleza de las voces y a las realidades extralingüísticas que existían en las islas. Los misioneros temían que los filipinos confundieran los significados y se generaran 
creencias paganas. En consecuencia, en este trabajo se estudiarán los métodos de traducción que llevó a cabo Domingo de los Santos en su obra lexicográfica para evitar estas confusiones de significado y da lugar a nuevos conceptos semánticos.

En cuanto a la nomenclatura, el autor selecciona voces españolas propias del léxico estándar de la lengua de Castilla. Por lo tanto, en el diccionario aparecen diferentes sustantivos, adjetivos y verbos españoles. También se ha de advertir que en la macroestructura hay adverbios, conjunciones e incluso partículas filipinas. Respecto a la inclusión de esta última categoría gramatical mencionada, cabe indicar que el tagalo era una lengua aglutinante, por lo tanto, diferentes partículas formaban sustantivos, adjetivos, verbos, etc. Este estudio se va a centrar en el tratamiento lexicográfico de las realidades filipinas, realidades americanas y españolas, en cuanto a estas últimas nos centraremos principalmente en las voces cristianas.

En este trabajo también se han estudiado algunas voces internas que aparecen en los artículos lexicográficos y que han cobrado interés para el estudio, debido a que se trata de léxico no lematizado pero clave para la enseñanza del español a nivel cultural. Esto ocurre con voces propias del léxico religioso Espiritu Santo o con el léxico político de la época como por ejemplo capitán.

Domingo de los Santos, como los demás misioneros, tuvo que tomar el papel de lingüista y afrontar los problemas adscritos a la normalización de aspectos como la ortografía, las variaciones léxicas o las unidades fraseológicas. El autor, de forma general, introduce los sustantivos en singular y en masculino. No obstante, en algunos casos usa el plural para lematizar los sustantivos: calabaz̧as, plátanos, aceitunas, chirimías, abejones, etc. Además de la flexión del plural, encontramos también la flexión morfológica para formar diminutivos, como por ejemplo pescadillo. En consideración a los verbos, el autor utiliza el infinitivo de forma sistemática.

Domingo de los Santos inserta unidades fraseológicas y quedan de forma lematizada dentro del diccionario como una entrada simple y ordenada alfabéticamente. Por ejemplo, rato ha, es decir, una forma impersonal que hoy sería hace rato y expresa temporalidad. 


\subsection{Aspectos MicRoESTRUCTURALES}

Domingo de los Santos también se basa en san Buenaventura (1994 [1613]) en la composición del artículo lexicográfico, por norma general reproduce la siguiente estructura:

- ENTRADA/VOZ: en la primera parte del diccionario, la entrada aparece en español, el autor presenta la transcripción fonética de las palabras en la escritura. Además, se distingue del resto del artículo lexicográfico.

- Traducción: Domingo de los Santos opta por diferentes métodos de traducción. El método más usado es el de la imposición de un equivalente tagalo. No obstante, otras veces usa un hispanismo adaptado o no a la fonética filipina. Otra opción elegida por el autor es crear una nueva palabra como veremos en algunos ejemplos de las voces religiosas y, en otras ocasiones, no traduce la palabra, sino que decide realizar una definición propia de la lexicografía monolingüe.

- MARCACiÓN fONÉTICA: todos los artículos la contienen. En cuanto a la representación fonética, cabe destacar que este diccionario presenta la transcripción fonética de las palabras en la escritura. Así mismo existe la marcación $\not p$ o $p c$. Según indica el autor en la advertencia al lector es muy importante, ya que establece la pronunciación de las palabras.

- DEFINICIÓN: las definiciones son por lo general aclaratorias propias y dan información semántica de los usos de las voces.

- Ejemplo tagalo - ESPAÑOl: este franciscano hizo una gran selección de ejemplos representativos de la intención evangelizadora del autor. En Lexicografía, los ejemplos “constituyen el contrapunto exacto de la definición, a la cual ilustran y en cierta manera complementan. Tienen, por una parte, carácter de testigos y, por otra, constituyen la mejor prueba de la validez y adecuación de la definición” (Fernández Sevilla, 1974, p. 78). De este modo, además de completar el significado de las palabras, podría evitar problemas de significado de voces desconocidas, además ayudaría a otros misioneros a trasmitir el evangelio en la lengua tagala. La traducción española aparece justo 
después del ejemplo tagalo, como en el caso anterior, sirve al lector como comprensión del significado de las palabras en contexto.

(1) Quemadura. Paso (pp) en cualquiera parte del cuerpo. Mahapdi yaring pasoco. Mucho escuece esta quemadura (Santos, 1794 [1703]: s.v.).

A continuación, el estudio se centrará en el tratamiento lexicográfico y métodos de traducción de los referentes de la cultura europea a las lenguas filipinas y de los específicos del catolicismo, además, se van a presentar algunas voces propias de realidades americanas. Igualmente, se estudiará el modo en el que Domingo de los Santos hizo un intento de renovar el significado de algunas realidades culturales filipinas.

\subsection{MÉTOdO LEXICOGRÁFICO}

\subsubsection{Tratamiento lexicográfíco de las realidades filipinas}

Domingo de los Santos (1794 [1703]) utiliza diferentes estrategias para presentar en su diccionario nuevas realidades propias de Filipinas.

A) Una de las estrategias más usada consiste en crear diferentes entradas de una voz española, que suele ser un hiperónimo, e introducir diferentes equivalentes para cada entrada:

(2) Arbol (sic). Putar (pp) cuyas hojas se comen. Mohaca nang dahonang putar (s.v.)

(3) Arbol (sic). Balobar (pp) largo y angosto, cuyas hojas tiernas se comen guisadas o en la olla. Dahon nang balobar (s.v.)

(4) Arbol (sic). Dita. (pc) medicinal, también sirve para algunas cosas, como para hacer guitarras (s.v.)

(5) Arbol (sic). Tuba. (pp) que la fruta sirve para matar pescado en el río. Dalaua ang tuba cong cahuy. S.e.f. tengo dos árboles de estos (s.v.)

(6) Bufalo (sic). Anuang. (pc) animal conocido son muy mansos los caseros. Los cimarrones son peores que toros de Xarama [sic] (Jarama) [...] (s.v.)

Domingo de los Santos, a través de esta práctica lexicográfica ofrece al lector una visión de la realidad filipina. El autor da una información descriptiva y, siguiendo a Moreno Moreno (2007, p. LXXI) usa la definición teleológica. De este modo, "recoge información sobre la naturaleza de las cosas en razón del fin a que se destinan". Algunos ejemplos de definición teleológica son:

(7) Yerva (sic). Sagumay (pp) amarilla con que hacen labores en las petacas. Humanapca nangsagumay. Busca de esta yerva [sic] (hierba) (s.v.) 
(8) Yerva (sic). Ticasticas. (pp) cria unas bolsillas, y en ella cuentas negras de que hacen rosarios (s.v.)

(9) Yerva (sic) Baling-uay. (pc) como bejuco, pero muy delgada. Las hojas son medicinales, y el cuerpo para amarrar cualquiera cosa (s.v.)

(10) Yerva (sic). Tala (pp) Muy olorosa. Suelen echarla en las Iglesias en las festividades (s.v.)

No obstante, este tipo de definición usada para describir las realidades filipinas alterna con la definición enciclopédica:

(11) Arbol (sic). Talisay. (pp) este cría una fruta a manera de piles, y porque cuando se le cae la hoja a dicho árbol, se le cae parte de la pluma a cierto género de gallos, llaman a tal gallo. Talisayin (s.v.)

(12) Fruta. Lansones. (pc) de la tierra; aunque los hay en pocas. Son muy dulces, aunque tienen poco que comer (s.v.).

(13) Fruta. Balingbing. (pc) ochavada, es buena. Hay la dulce y agria. Humanap ca nang balingbing. Busca fruta de esta (s.v.).

(14) Fruta. Sinangday. (pc) De sartén que hacen de harina y miel. Comain ca nang sinangday. Come fruta de sartén (s.v.).

En otras ocasiones realiza una definición aclaratoria propia, puesto que para él es importante resolver la vaguedad del término que funciona como entrada y determinar la proximidad semántica del equivalente tagalog.

(15) Yerva (sic). Libato (pp) Comestible. Mitaca nanglibato. Buscar yerva de esta (s.v.).

(16) Yerva (sic). Bacong. (pp) Es medicinal. Tapalanmo nangbacong. Ponle emplasto, de esta yerva (s.v.).

(17) Pajaro (sic). Pipit. Muy pequeñito (s.v.).

(18) Pajaro (sic). Coling. (pp) de buen canto (s.v.).

Es decir, el autor realiza un esfuerzo para poder aclarar los significados de los equivalentes tagalos, puesto que los lectores de esta obra, probablemente otros misioneros, no fueran conocedores del mundo del archipiélago.

B) Para introducir realidades filipinas no solo usa como entrada el hiperónimo, sino que también se sirve del hipónimo español y la definición sigue ajustándose a los patrones de una definición teleológica, enciclopédica o aclaratoria.

(19) Calabaza. Condol (pc) largas y delgadas de que hacen potaje. Magcondol ca sa Laguerta. 2. Act. ${ }^{3}$ Siembra calabazas largas en la Guerta (s.v.).

\footnotetext{
${ }^{3}$ En el diccionario aparecen marcas gramaticales, pero no de forma sistemática. Este trabajo se centra en la originalidad del autor como traductor y lexicógrafo de realidades distintas entre dos culturas. Además, como se ha explicado anteriormente, en esta etapa los diccionarios carecían de interés gramatical y era inservible para la enseñanza del español.
} 
(20) Calabazas. Tabayag (pp) grandes y silvestres, no sirven sino de echar en ellas agua o vino y semillas (s.v.).

(21) Plantanos (sic). Tondoc (pc) Grandes y de mucha sustancia (s.v.).

(22) Plantanos (sic). Ynambac, I, Calbonḡolo, galiyan, otonğan, sacsic, yniba, [...] (s.v.)

En el último ejemplo se aprecia que el autor decide no crear diferentes entradas para los distintos nombres que registra para la voz plantano [sic] (plátano) Por lo tanto, encontramos diferentes variedades lingüísticas de una voz tagala, ynambac que es el equivalente seleccionado por Domingo de los Santos. Esto mismo ocurre con la voz vino:

(23) Vino. Alac (pp) Nombre general. Matapang naalac. Vino fuerte. Nag aalacsila. Están haciendo vino. Anongynaalac ninyo? De qué hacéis vino? Alac saniyog., I, Sa sasa., I, Sa Castila. Vino de coco, de Nipa, o de Castilla (s.v.).

En la actualidad se conservan las dos variedades de vino procedentes de Filipinas: vino de coco y vino de Nipa.

C) Como se ha indicado anteriormente, Domingo de los Santos era un gran conocedor de la lengua tagala y, a su vez, de la cultura filipina. Esto queda claro en su obra lexicográfica. De hecho, para hacer alusión a nuevas realidades filipinas, en la definición de algunos artículos lexicográficos incluye información diatópica:

(24) Pescadillo. Ayonḡin (pc) Pequeño. Hay mucho en la laguna de Bay (s.v.).

(25) Pescadillo. Bacoli. (pc) estos se cogen en los ríos de los Tingues (s.v.).

(26) Pescadillo. Dilis (pp) del tamaño de un dedo, aunque delgados, cogen mucho cerca de Manila (s.v.).

(27) Pescado. Malasugui. (pp) De la mar de Mauban. Es muy precioso. Malasugui yati. Este es pescado precioso (s.v.).

(28) Pescado. Pantat. (pc) de los ríos de los montes, es muy suave. Mita ca nang pantat (s.v.).

(29) Pescado. Biya. (pp) Muy ordinario, cójase en los ríos y en la Laguna de Bay. Es blanco (s.v.).

El autor intenta diferenciar las distintas realidades a través de la explicación geográfica en la que él ha documentado estas nuevas especies.

D) En este diccionario también se ha encontrado que el autor usa una realidad española que guarda algún parecido con el equivalente tagalo. Este tipo de selección léxica debe quedar explícita en la definición. Por lo tanto, utiliza diferentes definiciones para dar a conocer que lo que realmente está 
describiendo es una realidad filipina y no española, en general usa una definición enciclopédica:

(30) Anis (sic). Sanqui. (pc) es de China y muy diferente, de el de Castilla, aunque tiene buen gusto. Nagsasangqui itons Sangley. 2. Act. Este Sangley vende anís (s.v.).

El autor usa el término español para determinar una voz, que como indica en la definición, es china. De hecho, en la actualidad se ha mantenido esta variedad de anís y lo denominamos en español: anís de la China. Además, indica que es diferente al de Castilla. No obstante, en este trabajo se deduce que el hecho de haber seleccionado esta voz española se debe a que comparte algunas características relacionadas con el color, el olor o el sabor.

En las siguientes líneas se va a estudiar pormenorizadamente cómo introduce esta información en el definiens. En primer lugar, se presentarán algunos ejemplos de definición sinonímica para dar significado a este tipo de realidades:

(31) Piñones. Pili. (pp) o almendras que todo es uno es fruta de la tierra, parecida en algo a la almendra y nada al piñón, salvo en el gusto, que tiene algún remedo (s.v.).

(32) Pajaro (sic). Pogo. (pp) como un pardal o gorrión de España (s.v.).

(33) Calabazas. Patola (pp) cohombros, tienen la cáscara verde encendida (s.v.).

(34) Bellota. Basacan (pc) como la de España y la comen los puercos (s.v.).

En el primer caso, usa el sinónimo almendras y utiliza un sintagma preposicional de la tierra para marcar que es una realidad de Filipinas. El autor realiza una aclaración en relación con la selección léxica, puesto que se parece a la almendra, pero sabe igual que el piñón a pesar de su diferenciación física. En las demás definiciones de las otras voces se usa la fórmula como la de España o como [...] de España, excepto en la voz calabazas puesto que se ha introducido directamente una voz española como sinónimo.

El tratamiento lexicográfico de las nuevas realidades a partir de entradas españolas también sigue otros patrones diferentes al anterior. De este modo incluyen la definición relacional de la tierra: 
(35) Turron (sic). Buc-hayo (pp) De la tierra. Bus-hayo naua. Ojalá hubiera turrón. Ypag buc hayo mo aco (s.v.).

(36) Higo. Tibig (pc) de la tierra. Comain ca nang tibig. Come higos (s.v.).

Además de definiciones teleológicas que indican el uso que los naturales les daba a las cosas que se describen en el diccionario:

(37) Añil. Tayom. (pp) una yerba con que tiñen de azul. Mita ca nang tayom. Busca añil,. Valan ytatayom dito sa maputi. I. P. no hay con que teñir de azul, esto blanco (s.v.).

(38) Amarillo. Barac. (pp) es una raíz con que se untan de amarillo (s.v.).

(39) Avanico (sic). Paypay (pc) con que echan viento. Nag paypaypaycang mag hapon diyan sa may saquit. 2. Activ. Todo el día estás echando viento con el avanico a ese enfermo (s.v.).

E) Otras veces directamente se adopta una palabra filipina a la fonética española como ocurre en:

(40) Champan (sic). Sampan. (pc) embarcación de Sangleyes: es chata. Nang sasampan. 2. Act. Dice hacerlo, y andar en él (s.v.).

F) Parece ser que los misioneros crearon diferentes neologismos para nombrar realidades filipinas. Domingo de los Santos (1794 [1703]) introdujo el neologismo morisqueta como entrada. Esta palabra nueva no la creó él como se mostrará a través de los datos recogidos de CORDE y el NTLLE:

(41) Morisqueta. Sinaing. (pp) Arroz cocido. Yhayinmo iyang sinaing. Saca o ofrece esa morisqueta (s.v.).

(42) Morisqueta. Maloto. (pp) Que llevan para comer en el camino. Comain tayo nang maloto. Comamos morisqueta... (s.v.).

(43) Morisqueta. Canin. (pp) Ordinario Pan de los naturales. Uala caming canin. S. r. f. No tenemos morisqueta (s.v.).

Según los datos extraídos en CORDE, esta voz se documenta entre 1611 y 1650 en una obra llamada El libro de las medicinas caseras, de fray Blas de la Madre de Dios, publicado en Ediciones de Cultura Hispánica (Madrid) por Francisco Guerra y María del Carmen Sánchez Téllez. En la ficha de CORDE se indica que el país de procedencia de esta obra es Filipinas.

Como se ha comentado anteriormente, fray Domingo de los Santos siguió a fray Pedro de San Buenaventura. En la obra de este último, se recogen tres entradas también, cuyos equivalentes son los mismos que recoge el autor de la 
obra que se está analizando. Sin embargo, los significados de la primera y tercera entrada cambian. Sí es cierto que en ambas se hace alusión a alguna comida propia de los naturales de aquellas islas, pero san Buenaventura en la primera entrada no especifica qué alimento es exactamente, es decir, indica que sinaing $^{4}$ es 'comida ordinaria de estos' y en la tercera acepción ${ }^{5}$ habla de 'arroz guisado', no de pan como hace el autor de esta obra.

Según el DLE (2014), en su segunda acepción, morisqueta es 'Arroz cocido con agua y sin sal, propio de Filipinas'.

Cabe destacar que morisqueta aparece por primera vez en la lexicografía académica en $1734(\mathrm{DA})^{6}$. No obstante, el significado que se recoge no corresponde con la realidad que se está analizando, sino que señala: “el ardid o treta propia de los Moros: lo que por translación se dice que cualquier acción con que se pretende engañar o burlar o despreciar a otro [..]”. Por lo tanto, hay que esperar a $1884(\text { DRAE })^{7}$ para que aparezca la definición que se conserva hasta en la última edición.

Por lo tanto, según los datos recogidos, a pesar de que en el siglo XVII se empezara a usar esta palabra originada, probablemente en Filipinas, no aparece en la lexicografía académica hasta el siglo XIX. En este trabajo se defiende que posiblemente fuera un neologismo, ya que, si se consideran los datos históricos, los sultanatos islámicos surgieron en el siglo XIV en Filipinas (Fernández Martínez, 2000, p. 72). La influencia musulmana, debido a la ruta comercial, pasó por Mindanao, Bisayas y Luzón (Prieto Lucena, 2000, p. 85). Esta misma autora indica que estos mercaderes dejaron su influjo religioso en algunas zonas de las islas, especialmente en Mindanao (2000, p. 102).

Por lo tanto, los españoles -después de haber convivido con musulmanes durante tantos siglos, conocer sus costumbres y saber que los árabes llegaron

\footnotetext{
${ }^{4}$ Equivalente también usado por Domingo de los Santos. Ver ejemplo 48.

${ }^{5}$ El equivalente es el mismo que usa Domingo de los Santos. Sin embargo, los significados cambian.

${ }^{6}$ Diccionario de Autoridades.

${ }^{7}$ Diccionario Usual.
} 
a Filipinas- usaron esta palabra para referirse a una comida que les recordaba a alguna propia de los moriscos castellanos.

\subsubsection{Tratamiento lexicográfico de realidades americanas}

Domingo de los Santos (1794 [1703]) introduce voces procedentes del Nuevo Mundo americano, como la voz americana jicamas:

(44) Xícamas (sic). Singcamas (pc) A modo de nabos redondos, comense crudas, y son muy dulces (s.v.).

Usa como definidor una locución adverbial 'a modo de' para definir el significado de jícamas e introducir un equivalente español que sirve como sinónimo de la entrada. De esta manera, realiza una definición enciclopédica.

Los españoles en el continente americano decidieron usar voces europeas para nombrar realidades americanas. Esto ocurre con las voces: tabaco, balsamina o piña que se encuentran en la nomenclatura:

(45) Tabaco. Tabaco (pp) Yerva (sic) medicinal (s.v.).

En su diccionario incluye la voz hispana tabaco para nombrar una realidad americana. Así mismo, introduce esta nueva realidad al léxico tagalo a través de una definición perifrástica: 'género propio’ (hierba) junto a la 'diferencia específica’ (medicinal).

(46) Piña. (pc) Fruta de México: que acá no la había, ya hay muchas. Desengaña la comida (s.v.).

En este trabajo se deduce que realmente se hace alusión a la fruta ananás que era procedente de América y tiene forma de piña. Además, en la parte definitoria se indica que es una realidad nueva en Filipinas. Por lo tanto, los colonos fueron los que trasportaron esta planta al archipiélago filipino.

\subsubsection{Tratamiento lexicográfico de voces españolas}

Los misioneros hicieron el esfuerzo de encontrar equivalencias para las voces españolas. No obstante, no siempre esto fue posible, por lo tanto, tuvieron que llevar a cabo diferentes técnicas de traducción. Siguiendo a Sueiro Justel (2002) la dificultad del quehacer lexicográfico radica en traducir realidades desconocidas en otros pueblos y no hallar equivalencias, asimismo, la práctica lexicográfica misionera se encuentra 
con otro problema que consiste en poner dos culturas distintas sin tergiversar el sentido. Zimmernann (2004, p. 108) también afirma que el problema de la traducción y denominación de realidades religiosas se debía a las diferencias que existen entre ambas lenguas.

En este apartado se va a estudiar el tratamiento lexicográfico de las voces cristianas y otras propias del léxico estándar. Tras haber realizado un vaciado léxico de las voces cristianas, se ha obtenido la siguiente clasificación ad hoc: a) equivalentes léxicos, b) hispanismos, c) creaciones léxicas, y d) extensiones semánticas.

A) Equivalentes léxicos: dios, iglesia, ofrenda, alabar, cielo, entre otras. Desde una perspectiva de lingüística contrastiva y traductológica simplista, los misioneros consiguieron descifrar algunas equivalencias léxicas en las lenguas indígenas (Zimmermann, 2005, p. 120). No obstante, esta intención de codificación no acompañaba al objetivo evangelizador del clérigo, puesto que podía llevar a confusiones a la hora de transmitir el significado a los receptores, que son los habitantes de la provincia de san Gregorio. El equivalente bathala, como indica Fernández Rodríguez (2014, p. 287), procede del sánscrito. Asimismo, cabe destacar que según Gutiérrez (1992) Bathala era creador de todas las cosas y que vivía aislado de los hombres, de tal modo que los filipinos no se atrevían a dirigirse directamente a él y por este motivo, entraban en contacto con los anitos que eran otros espíritus.

Domingo de los Santos no solo encuentra su equivalente léxico, sino que a través del artículo lexicográfico usa diferentes marcas para advertir que se ha realizado una sustitución semántica:

(47) Dios. Bathala (pp). De creer es, llamaban así a Dios Nuestro Señor, pues decían. Ang Bathala nangmaycapal sa lahat. El Dios criador, o hacedor de todas las cosas. Y esto, a diferencia de los ídolos, que a unos llamaban Anito y a otros, Lucha, id est Estatua. Y cada uno servía para una cosa; y así decían. Magpabathala ca. Déjate a la voluntad de Bathala (s.v.).

Después del intercambio de significantes, realiza una definición enciclopédica, ya que hace una explicación detallada de una realidad extralingüística, en este caso trata las creencias de la cultura filipina. A través de la fórmula 'de creer es', 
el autor explica el uso de bathala puesto que los tagalos creen en él porque es 'Dios Nuestro Señor'. Domingo de los Santos introduce este equivalente porque Bathala tiene el mismo significado que el dios cristiano, esto es, dios es criador y hacedor de todas las cosas. Además, explica la creencia politeísta de Filipinas.

Otra palabra muy importante en el cristianismo que ha sido traducida por equivalente tagalo es iglesia. El misionero ha utilizado el equivalente 'simbahan' que era un lugar sagrado para los filipinos donde realizaban los sacrificios. Por lo tanto, a través de la estrategia del uso del equivalente, se ha realizado una sustitución semántica de la realidad.

También ocurre lo mismo con el lema ofrenda. Nos vamos a detener en esta entrada para analizar las fórmulas lexicográficas que usa con el fin de dejar claro la sustitución semántica que ha habido:

(48) Ofrenda. Hayin. (pp) que uno ofrece a Dios [...] (s.v.)

Después de haber ofrecido varios ejemplos de equivalencia léxica, cabe señalar que el religioso se cerciora de que seleccionar un significante para otro significante es insuficiente para poder llevar a cabo su misión como franciscano en aquellas tierras. Por lo tanto, intenta cambiar conceptualmente los términos tagalos a partir de la definición donde da explicaciones o atribuye marcas semánticas para aclarar el sentido religioso de esas voces.

B) Hispanismos: altar, arzobispo, incensario, infierno, misa, misal, ostia, padre, purgatorio, cuaresma, santo, vigilia, etc.

Los misioneros temían que sus traducciones pudieran provocar confusiones de significado. Además, tal y como apunta Fernández Rodríguez (2009, p. 269), los clérigos muestran una gran preocupación por el rigor y la precisión semántica para no dar lugar a malentendidos religiosos y culturales. Por lo tanto, el uso de hispanismos como estrategia traductológica de voces cristianas no es extraño. La elección de este método de traducción no solo viene motivada por el temor de no transmitir el significado religioso, sino que 
también se puede deber al vacío léxico que existe debido a la introducción de una nueva realidad cultural a otra cultura totalmente distinta.

(49) Padre. Pare (pp) religioso o misionero, en lugar de Padre, quitanle la D y dicen Pare o Pari. Otos nang Pare. Mandato del Padre. Nagmimisa ang Pare. El Padre está diciendo missa (s.v.).

Domingo de los Santos introduce el hispanismo actualizándolo conforme a la fonética filipina, ya que como indica él, los tagalos eliminaban la $d$ y se realiza una restructuración silábica. Además, ofrece una definición sinonímica en la que el misionero acude a dos cuasisinónimos 'religioso' y 'misionero'. De esta forma, aclara al receptor del diccionario, probablemente otro religioso, que se usa el hispanismo pare cuando se quiera hacer alusión a un religioso o a un misionero.

En otras ocasiones, no se hace una aproximación de significado, simplemente se remite al hispanismo y se conserva el ejemplo:

(50) Missa (sic). Missa. (pp) Nagmi Missa ang Padre. 2. act. El padre está diciendo missa. Casangcapang ypagmi Missa. 2.P. Recado con que se dice Missa. Pinagmimissahan. El Altar, y la gente a quen se dice. [...] (s.v.).

En relación con los hispanismos, el autor realiza una definición verdadera, esto es, contienen género próximo y diferencia específica, aunque esta práctica se corresponde más con el quehacer lexicográfico monolingüe:

(51) Rossario (sic). Couentas (pc) Para rezar. Bacsahin mo ang couentas mo. Ponte al cuello el rossario. Manḡointas ca. Roza el rossario (s.v.).

Se ha utilizado el género próximo 'cuentas' y la diferencia específica es 'para rezar', es decir, el misionero no ha utilizado un método de traducción, sino que ha definido la voz rossario.

C) Creación léxica: la creación de ciertos neologismos dentro de una comunidad de hablantes se genera debido a la necesidad de poder expresar nuevas realidades. En el caso de esta obra realizada por un misionero, se trata de neología referencial, puesto que usa este recurso de traducción para denominar nuevos conceptos, principalmente culturales. 
Domingo de los Santos no solo realiza innovaciones léxicas a través de la inclusión de afijos a raíces tagalas, sino que también lo hace con raíces españolas. En cuanto a los neologismos creados a partir de las raíces tagalas tenemos: yniyna (s. v. madrina), yna ama (s. v. padrino), panalangin (s. v. oración), catotobo (s. v. ángel de la guarda) pananangpalataya (s. v. fe), etc. En relación con las raíces españolas tenemos pagpapa confesala (s. v. confesionario), magpapamisa (s. v. monacillo) y casantosan (s. v. santidad). En primer lugar, vamos a dar algunas muestras de creación léxica en voces tagalas:

(52) Madrina. Yniyna (pc) De bautizo o de confirmación (s.v.).

Yniyna ha sido creada a partir de la raíz yna que significaba 'madre' en Manila. Cabe destacar que se ha introducir también el prefijo $y$ - que se usaba para nombrar a la madre en algunos pueblos filipinos. Se ha de tener en cuenta que el neologismo tagalo creado para padrino es yna ama. En este caso se ha introducido una nueva voz a través de composición: sust. + sust., esto es yna que significaba 'madre' junto al lexema ama que en tagalo significaba 'padre'.

Otro ejemplo de creación léxica a través de un lexema tagalo ocurre con la traducción de la locución nominal ángel de la guarda:

(53) Angel (sic). Catotobo. (pp) de guarda. Llamanle así por cuanto le da Dios a cada uno el ángel de la guarda; y ellos presumen, con su modo rústico, que el ángel nace con el hombre; que esto significa tobo que es nacer las plantas. Ang catotobo cong ángel. El ángel de mi guarda (s.v.).

El misionero ha creado este neologismo a través de la composición. Ha unido los lexemas catoto que en español significa 'amigo' y el lexema tobo que significa 'ángel que nace con el hombre'. Por lo tanto, catotobo es el ángel de la guarda cristiano, puesto que el religioso ha creado esta palabra para que no se confunda con la creencia filipina de que el ángel nace con el hombre. Para la tradición cristiana, dios es el encargado de darle un ángel de la guarda a cada uno de los hombres.

A continuación, se analizarán algunos neologismos creados a partir de raíces españolas: 
(54) Monacillo. Magpapamisa. (pp) que ayuda a Misa. Ualan magpapamisa saaquin. No hay monacillo, o Acolyto que me ayude a Misa (s.v.).

Se ha formado una nueva palabra a partir de dos lexemas españoles papa y misa a los cuales se les ha unido el prefijo tagalo mag-.

En el caso de pagpapa confesala (s. v. confesionario) se ha creado una locución nominal a partir de pagpapa, se le ha añadido un prefijo tagalo pag al lexema español papa y la voz confesala que es un compuesto híbrido formado por la palabra confesar y sala que significa 'pecado o culpa'.

D) Extensión semántica: bautizar, signarse, santiguarse, buerta, pecado y ángel de la guarda ${ }^{8}$.

Como se verá a continuación a través de los ejemplos, en este diccionario encontramos neologismos semánticos, esto ocurre, en palabras de Casado Velarde (2015, p. 98): "cuando un signo de la lengua adquiere un nuevo significado procedente de otra lengua". En esta obra lexicográfica se han encontrado similitudes conceptuales entre dos términos, uno español y otro filipino y se han creado nuevas acepciones por extensión semántica del significado original.

Cabe señalar que la extensión semántica de bautizar ya ha sido tratada por otros autores como Fernández Rodríguez (2014, p. 284). Esta autora explica que la voz biyang significaba en su origen 'mojar' en tagalo. Seguramente no resultara complicado implantar un significado nuevo a este significado tagalo, ya que, según Gutiérrez (1992, p. 119) los filipinos pensaban que el bautismo tenía propiedades curativas. No obstante, debido a esta creencia, los misioneros temían que este sacramento adquiriera un significado pagano y se llegara a convertir en un rito mágico y no cristiano.

Otro ejemplo de extensión semántica ocurre en la voz yariangtanda (s.v. signarse). Esta voz significaba literalmente 'esta señal' (García Medall, 2013, p. 57). Sin embargo, por extensión semántica y por necesidades cristianas,

\footnotetext{
${ }^{8}$ En el diccionario aparece una nueva entrada de ángel de la guarda en este caso su traducción se debe a una extensión semántica de tanor que tenía en tagalo diferentes significados: pastor, custodiado o guarda. Por lo tanto, la acepción cristiana deriva del campo semántico de protección, puesto que el ángel de la guarda se encarga de proteger al hombre.
} 
también adquirió el significado de 'hacerse señal de la cruz', por lo tanto, yariangtanda es el equivalente tagalo de persignarse, santiguarse y signarse.

Por último, se va a hacer alusión a la voz tagala halamanan (s. v. guerta), cuyo significado es 'huerta de árboles’. A este signo lingüístico tagalo se le ha añadido un significado cristiano 'paraíso'. Esto se debe a que en la Biblia se usa la palabra huerto para referirse al paraíso, es decir, al jardín del Edén.

\section{CONCLUSIONES}

Tras el análisis de la obra lexicográfica ofrecida, se han comprobado que los objetivos de este trabajo han sido alcanzados gracias al estudio exhaustivo del material léxico, organización del diccionario y del tratamiento lexicográfico de las diferentes voces localizadas tanto en la macroestructura como en la microestructura. De este modo, se ha indagado sobre los posibles modelos lexicográficos y la originalidad del autor como traductor y transmisor de realidades de dos mundos totalmente opuestos.

Cabe destacar que Domingo de los Santos realiza auténticos esfuerzos para ejercer como traductor en Filipinas. Su ardua labor se refleja en la introducción de nuevas realidades filipinas y en los diferentes métodos de definición para poder ser transmisor real de aquellas tierras. Asimismo, incluye realidades americanas ya conocidas desde la primera expedición en el continente americano.

Siguiendo a Zimmermann (2005) y tras haber analizado esta obra, se piensa que en Filipinas se generó una transculturación recíproca. La transculturación de los individuos de un lado conlleva la transculturación de individuos de otro lado: de este modo se han adquirido realidades filipinas como paipay, vino de Nipa, anís de la China, etc. Cabe advertir que además de las voces propias del cristianismo, también se han transmitido algunas realidades españolas en Filipinas como: chinelas, comedia, fiscal, capote, castañetas, cidras, chirimías, entre otras.

Como conclusión queda resaltar la importancia que tuvo Domingo de los Santos como traductor y continuador de una vertiente lexicográfica bilingüe empezada con el diccionario hispano-tagalo de Pedro de San Buenaventura (1994 [1613]). Por lo tanto, sería interesante seguir estudiando la continuación de esta labor a través de otros 
autores de esta y distintas órdenes con el fin de ahondar sobre el proceso de las teorías traductológicas, conocer la política de préstamos y las voces que seguimos manteniendo en la actualidad gracias a las expediciones que tuvieron lugar en Filipinas.

\section{BIBLIOGRAFÍA}

Alvar Ezquerra, M. (2018). Biblioteca Virtual de la Filología Española (BVFE): directorio bibliográfico de gramáticas, diccionarios, obras de ortografía, ortología, prosodia, métrica, diálogos e historia de la lengua [en línea] $<$ www.bvfe.es> [18/04/2018].

Casado Velarde, M. (2015). La innovación léxica en el español actual. Madrid: Editorial Síntesis.

Fernández Martínez, V. M. (2000). La prehistoria de las islas filipinas, en L. Cabrero (Coord.), Historia General de Filipinas. Madrid: Ediciones de Cultura Hispánica, pp. 47-76.

Fernández Rodríguez, R. (2014). Traducción de términos religiosos en los vocabularios filipinos (1565-1800), en O. Zwartjes, K. Zimmermann y M. Schrader-Kniffki (Eds.), Missionary Linguistics V/Lingüistica misionera V: Translation theories and practices, Amsterdam/Philadelphia: John Benjamins, pp. 273-294.

Fernández Rodríguez, R. (2009). El Calepino Ilocano (c.1797) del P. Vivar: Innovaciones lexicográficas y política traductora, en O. Zwartjes, R. Arzápalo Marín, T. C. Smith-Stark (Eds.), Missionary Linguistics IV/Lingüistica misionera IV : Lexicography. Amsterdam/Philadelphia: John Benjamins Publishing, pp. 249-272.

García-Medall, J. (2004). Notas de lexicografia hispanofilipina: El Bocabulario de lengua bisaya, hiligueyna y haraya de la isla de Panay y Sugbu y para las demas islas, de fray Alonso de Mentrida, OSA (c.1637), en O. Zwartjes y E. Hovdhaugen (Eds.), Missionary Linguistics/Lingüistica misionera, Amsterdam/Philadelphia: John Benjamins, pp. 201-232.

García-Medall, J. (2007). La traducción codificada: las artes y Vocabularios hispano-filipinos (1610-1910), en Hermèneus. Revista de Traducción e Interpretación, 9, pp. 117-144.

García-Medall, J. (2013). En torno a los primeros préstamos hispánicos en Tagalo. Cuaderno internacional de estudios humanísticos, 19, 51-66.

Gómez Platero, E. (1880). Catálogo biográfico de los religiosos franciscanos de la provincia de San Gregorio Magno de Filipinas desde 1577 en que llegaron losprimeros a Manila hasta los de nuestros días, Manila Imp. del Real Colegio de Santo Tomás.

Moreno Moreno, M. a Á. (2005). Obras lexicográficas, instrumentos para la evangelización Franciscana (ss. XVI-XIX), en M. C. Cazorla Vivas; N. M. Contreras Izquierdo; M. á. García Aranda y M. Á. Moreno Moreno (Coords.), Estudios de Historia de la Lengua e Historiografía Lingüistica. Actas del III Congreso Nacional de la AJIHLE (Jaén, del 27 al 29 de marzo de 2003) (pp. 329-340). Madrid: Compañía Española de Reprografía y Servicios.

Moreno Moreno, M. á. (2007). Estudio preliminary edición del Diccionario de arabismos de Diego de Guadix, Jaén: Servicio de Publicaciones de la Universidad.

Moreno Moreno, M. Á Á. (2016). El trabajo lexicográfico y de traducción en el Diccionario de romance en lengua subguana de Fr. Francisco Encina (1715-1760). Anuario de Letras. Lingüistica y Filología, 4 (2), pp. 205244. 
Prieto Lucena, A. M. (2000). Etnohistoria de Filipinas. En L. Cabrero (Coord.) Historia General de Filipinas, Ediciones de Cultura Hispánica, Madrid, pp. 77-118.

Quilis, A. (1982). El Arte y Reglas de la lengua tagala. Nueva Revista de Filología Hispánica, 31/1, pp. 1-24.

Quilis, A. (1997). Datos para la historiografía lingüística filipina, en R. Escavy y Zamora, J. M. Hernández Terrés, E. Hernández Sánchez y M. ${ }^{a}$ Isabel López Martínez (Eds.), Homenaje al profesor A. Roldán Pérez, II, Murcia, España: Universidad de Murcia, pp. 471-495.

Rafael, V. L. (1993). Contracting Colonialism: Translation and Christian Conversion in Tagalog society under early Spanish rule. Durham and London: Duke University Express.

Real Academia Española. Corpus diacrónico del español (CORDE) [en línea], Disponible en $<\underline{\text { http://corpus.rae.es/creanet.html }>\text { [05/05/2018] }}$

Real Academia Española. Nuevo tesoro lexicográfico de la lengua española (NTLLE), [en línea], $<\underline{\text { http://buscon.rae.es/ntlle/SrvltGUILoginNtlle }>\text { [05/05/2018] }}$

San Buenaventura, P. de. (1994). Vocabulario de la lengua tagala, el romance castellano puesto primero. Primera y segunda parte. Por fray Pedro de San Buenaventura, en la noble villa de Pila, por Thomas Pinpin y Domingo Loag, año de 1613. Ed. facsimilar, Valencia: París-Valencia.

Santa Inés, F. D. (1892 [1676]). Crónica de la Provincia de San Gregorio Magno de religiosos descalzos de NSP San Francisco en las Islas Filipinas, China, Japón. Manila: Tipo-litografía de Chofre.

Santos, D. de los. (1794 [1703]). Vocabulario de la lengua Tagala, primera y segunda parte: en la primera, se pone primero el Castellano, y despues el Tagalo: y en la segunda al contrario, que son las rayzes simples con sus accentos. N(uestra) $\mathrm{S}$ (eñora) de Loreto, Sampaloc, por el Hermano Baltasar Mariano.

Sueiro Justel, J. (2002). La enseñanza de idiomas en Filipinas (siglos XV I-XIX), A Coruña: Toxosoutos.

Sueiro Justel, J. (2003). Historia de la lingüistica española en Filipinas (1580-1898), Lugo: Axac.

Zamora, P. Fr. E. (1901), Las corporaciones religiosas en Filipinas, Valladolid: Imprenta y Librería Religiosa de Andrés Martín.

Zimmermann, K. (2004). La construcción del objeto de la historiografía de la lingüística misionera, en O. Zwartjes y E. Hovdhaugen (Eds.), Missionary Linguistics/ Lingüistica misionera, John Benjamins, Amsterdam/Philadelphia, pp. 7-32.

Zimmermann, K. (2005). Traducción, préstamos y teoría del lenguaje: La práctica transcultural de los lingüistas misioneros en el México del siglo XVI, en O. Zwartjes y C. Altman (Eds.), Missionary Linguistics II/Lingüistica Misionera II: Ortography andphonology, Amsterdam/Philadelphia: John Benjamins, pp. 107-136. 